\title{
On quartet interactions in the California Current system
}

\author{
L. M. Ivanov ${ }^{1}$, C. A. Collins ${ }^{1,2}$, and T. M. Margolina ${ }^{1}$ \\ ${ }^{1}$ Naval Postgraduate School, Monterey, California, USA \\ ${ }^{2}$ Moss Landing Marine Laboratories, Moss Landing, California, USA \\ Correspondence to: L. M. Ivanov (lmivanov@nps.edu) \\ Received: 31 January 2014 - Published in Nonlin. Processes Geophys. Discuss.: 20 February 2014 \\ Revised: 22 July 2014 - Accepted: 22 July 2014 - Published: 29 August 2014
}

\begin{abstract}
Sea surface height (SSH) altimetry observations for 1992 to 2009 off California are used to show that observed quasi-zonal jets were likely driven by near-resonance interactions between different scales of the flow. Quartet (modulational) instability dominated and caused non-local transfer of energy from waves and eddies to biannual oscillations and quasi-zonal jets. Two types of quartets were identified: those composed of scales corresponding to (a) quasi-zonal jets, annual and semiannual Rossby waves and mesoscale eddies, and (b) biannual oscillations, semiannual Rossby waves and mesoscale eddies. The spectral centroid regularly shifted into the domain of low-order modes. However, the spectrum of SSHs does not demonstrate a power behavior. This says that the classical inverse cascade is absent. For a case with bottom friction, quartet instability required the existence of a certain level of dissipativity in the flow.
\end{abstract}

\section{Introduction}

In recent years, there has been growing recognition of the existence of persistent quasi-zonal jets (QZJs) in the midlatitude atmosphere as well as in the atmospheres of gaseous planets (an intensive review of such QZJs is given by Baldwin et al., 2007). These jets were clearly detected in the atmospheres of planets and the Earth because they were intense and had a clear signature in cloud systems, although the mechanisms of their generation were probably different. This is not the case for the Earth's oceans, because oceanic QZJs are latent (i.e. masked by more intense non-zonal flows), and can only be extracted from observations and model results by statistical procedures. Therefore, the role of QZJs in ocean dynamics and the nature of these jets are not clear, due to their weak signal. The principle question here is which physical mechanism(s) can generate the QZJs.

A number of the generation of QZJs includes modulation instability (as an example, see Connaugton et al., 2010), $\beta$ plume (Centurioni et al., 2008), inverse cascade (Galperin et al., 2010), and others. Most results were derived from analysis of two-dimensional models of QZJs that yielded useful analytical results (Loesch, 1977; Newell, 1969; Rhines, 1975; Vallis and Maltrud, 1991; Nadiga, 2006; Galperin et al., 2010; Connaugton et al., 2010; Wang et al., 2012, 2013, among others). However, these results were obtained for simplified jet models, and it is difficult to use these models to detect mechanisms of oceanic jet generation that might exist in nature.

Srinivasan and Young (2012) demonstrated that zonal jets could be reproduced with a linear theory, and did not rely upon the existence of an inverse cascade. The results presented here do not agree with Srinivasan and Young (2012) because in our opinion, nonlinearity played a very important role in the jet generation; therefore, results were not compared to those obtained by Srinivasan and Young (2012).

Alternative hypotheses based on three-dimensional models have been proposed to explain mechanisms of QZJ generation; see Hristova et al. (2008), Berloff et al. (2009, 2011), Tanaka and Akitomo (2010), Wang et al. (2012) and Qiu at al. (2013), among others. For example, Hristova et al. (2008) hypothesized that QZJs observed in the eastern region of subtropical gyres may be related to radiating instabilities of eastern boundary currents.

Connaugton et al. (2010) have suggested using a modulational instability concept for explanation of quasi-zonal jets observed in the ocean and atmosphere. Weak, longwave modulations of a periodic nonlinear wave train can grow exponentially under certain conditions. This is known 
as the modulational instability, and was first identified by Benajm and Feir (1967) for surface gravity waves on deep water. Modulational instability is a ubiquitous instability in nature and is not restricted to hydrodynamics. It also occurs in plasma physics, electrodynamics and nonlinear optics (Kharov and Ostrovsky, 2009). Modulational instability is one of the mechanisms for generating zonal flows in magnetically confined plasmas and in planetary atmospheres and oceans (Onishenko et al., 2008, Connaughton et al., 2010 and others).

Connaugton et al. (2010) found that, in a two-dimensional case for very weak primary waves, the unstable waves were close to being in three-wave resonance with the primary waves. They have revised the linear theory of Gill (1974) using a four-mode truncation, and have emphasized the role of the carrier amplitude/nonlinearity, the role of the deformation radius and the role of resonant wave interactions in the case of a stronger nonlinear carrier wave.

Ivanov et al. (2010) demonstrated that annual and semiannual Rossby waves could be interpreted as weak nonlinear waves because their nonlinearity parameters changed by as many as several units. They used a zonal component of propagation speed (that varied from zero to several $\mathrm{cm} \mathrm{s}^{-1}$ ), the wave steepness, $q(0.1-0.2<q<2-3$; the steepness was less than 1 for a considerable part of the wave evolution time), and the length of spatial phase coherence for detection of different regimes of Rossby wave evolution. It follows that nonlinear interactions between these waves should be studied with a view to understanding whether and how they might influence quasi-zonal jets.

Following Connaugton et al. (2010), we will study the weakly nonlinear stage of the modulational instability $(q<$ 1) and demonstrate that quartet interactions can generate quasi-zonal jets. From the theoretical point of view, these zonal jets subsequently become unstable and acquire the interesting two-dimensional structure of a double (Karmanlike) vortex street, which should be more stable than a plane parallel shear flow with the same zonal profile (McWilliams, 2006).

In the present paper, modulational instability is identified through the existence of quartet interactions when a Rossby wave with one year or semiannual periodicity is considered a carrier wave. This study analyzes sea surface height (SSH) observations off California, and shows that

a. mesoscale flow off California was driven by nearresonance interactions between flow scales. Four-wave near-resonance interactions between spatial scales dominated and formed specific structures of the mesoscale flow, including a combination of QZJs, biannual oscillations, annual Rossby waves, semiannual Rossby waves and mesoscale eddies of smaller scales.

b. the SSH signal was transferred from Rossby waves (RWs) and mesoscale eddies (MEs) to lower-frequency currents (biannual oscillations (BAOs) and QZJs). The existence of two different quartets was found: QZJ annual RWs semiannual RWs-MEs and BAOs semiannual RWs-MEs of two different scales. However, for both these quartets, the SSH signal was pumped from faster to slower scales, i.e., from annual and semiannual currents to biannual oscillations and quasi-zonal jets.

c. QZJ (or BAO) generation by modulational instability required the existence of a certain level of dissipativity in mesoscale flows. Dissipativity played an important role, because it determined a number of possible nearresonance interactions as well as the latency of observed QZJs.

In general, the dimensionality of analyzed QZJs cannot be based on the analysis of SSHs only. However, QZJs are shown to be dissipative structures, and their generation was the result of a self-organization process. Following Nicolis and Prigogine (1977), dissipative structures are defined as non-equilibrium thermodynamic systems that generate order spontaneously by exchanging energy with their external environment. Self-organization is the generation of regular structures in far-from-equilibrium states of a dissipative system due to interactions of the elements that make up the system (Nicolis and Prigogine, 1977).

The remainder of the paper is organized as follows. Section 2 describes the data and methods used. A system of jets (waves and eddies reconstructed from AVISO products) is discussed in Sect. 3. Robustness and repetition of QZJ generation are demonstrated in Sect. 3. Sections 4 and 5 include calculation of near-resonance and resonance interactions off central California. The re-distribution of surface elevation between different scales and scales dominating the surface elevation are discussed in Sect. 6. Although Sect. 6 is simply a kinematic analysis, it is shown that energy was pumped from Rossby waves and mesoscale eddies to QZJs and BAOs. The role of dissipativity in the process of energy re-distribution is discussed in Sect. 7. Section 8 shows how different scales forming quasi-zonal jets and biannual oscillations were grouped into separate quartets. Conclusions are given in Sect. 9.

\section{Data and methods}

The SSH anomaly field was produced by the AVISO (Archiving Validation and Interpretation on Satellite Data in Oceanography) project for the period from 10 October 1992 to 23 May 2009 (Collecte Localisation Satellites, 2006). To understand the spatio-temporal complexity of altimetry signals, a double spectral approach (DSA) (Ivanov and Collins, 2009) was applied to the analysis of the SSH anomaly field. Following this approach, the SSH anomaly field $\eta(\boldsymbol{x}, t)$ is 
represented by

$\eta(\boldsymbol{x}, t)=\sum_{m=1}^{M} a_{\mathrm{m}}(t) \Psi_{\mathrm{m}}(\boldsymbol{x})$,

where $\boldsymbol{x}=(x, y)$ is the position vector in a real space, and $\Psi_{\mathrm{m}}$ are basis functions (or M-modes that extend the classical Fourier polynomials to nonrectangular basins) calculated as in Ivanov and Collins (2009). Note that $\Psi_{\mathrm{m}}(\boldsymbol{x})=\sum_{l=1}^{L} c_{l \mathrm{~m}} \sin \left(k x / L_{x}\right) \sin \left(k y / L_{y}\right)$ only for a rectangular basin. Here, $L_{x}$ and $L_{y}$ are horizontal basin scales, $k=2 \pi / l$. Figure 1 shows the structure of several basis functions for the observational region. About 1000 basis functions were used to represent SSH with less than $1 \%$ rmse. Different combinations of basis functions formed currents that had different spatial scales.

The spectral coefficients $a_{\mathrm{m}}$ were then analyzed using a discrete wavelet transform as

$a_{\mathrm{m} j}(t)=\sum_{p=1}^{\infty} b_{\mathrm{m}, j, p} T_{j, p}(t)$,

where $a_{\mathrm{m} j}$ is an approximation of $a_{\mathrm{m}}$ with resolution $j$, $T_{j, p}=1 / 2^{j / 2} \varphi\left(2^{j} t-p\right)$, and $\varphi$ is the father wavelet. Finally, results were summed around selected timescales $j$ using Eq. (1).

A Debaucher sixth-order wavelet transform was used in this study. This allowed for the introduction of six frequency bands (FBs): (1) 1 month $\left(\omega \approx 2 \times 10^{-5} \mathrm{rad}\right)$ to 2 months $\left(\omega \approx 4 \times 10^{-5} \mathrm{rad}\right.$ ) (referred to as the mesoscale eddy II (ME-II) band), (2) 2 months $\left(\omega \approx 4 \times 10^{-5} \mathrm{rad}\right)$ to 4 months $\left(\omega \approx 8 \times 10^{-5} \mathrm{rad}\right)$ (ME-I band), (4) 4 months $\left(\omega \approx 8 \times 10^{-5} \mathrm{rad}\right)$ to 8 months $\left(\omega \approx 16 \times 10^{-5} \mathrm{rad}\right)(\mathrm{semi}$ annual Rossby waves or SARW band), (5) 8 months $(\omega \approx$ $\left.1.6 \times 10^{-6} \mathrm{rad}\right)$ to 18 months $\left(\omega \approx 3,6 \times 10^{-6} \mathrm{rad}\right)$ (annual Rossby waves or ARW band), (6) 18 months $(\omega \approx$ $\left.3,6 \times 10^{-6} \mathrm{rad}\right)$ to 36 months $\left(\omega \approx 7.2 \times 10^{-6} \mathrm{rad}\right)$ (biannual oscillations or BAO band), and (7) longer than 36 months $\left(\omega \approx 7.2 \times 10^{-6} \mathrm{rad}\right.$ ) (quasi-zonal jets or QZJ band). Coherent mesoscale structures (jets, propagating waves and eddies) were selected within these six frequency bands and for a spatial spectral band limited by the 30th (lower boundary) and 250th (upper boundary) M-modes.

Calculations should be robust for the choice of upper and lower boundaries: the results did not change when the upper boundary of the band was moved toward higher-order modes, but changed weakly when the lower boundary was moved to the 15 th mode. As discussed in Ivanov et al. (2010), modes from 1 to 29 were responsible for large-scale seasonal variability only. Rossby waves with periodicities from 4 months to 18 months cannot contain these modes, because this part of the spectrum demonstrates no movements westward and therefore was not included in the analyses.
Similarly, variations in observational sampling should not affect coherent (long-lived) structures. The length of observational sampling was reduced 4-6 times without changing the structure of quasi-jets. This was a consequence of using the Debaucher wavelet transform for the spectral representation of spectral coefficients $a_{\mathrm{m}}$.

Note that we are going to analyze wave modulation instability in an (M-mode, $\omega$ ) space, although the classical approach; see for example, (Zakharov and Ostrovsky, 2009) uses a $(k, \omega)$ space. The use of an M-mode makes the physical interpretation simpler, because $M$ spectrums are one dimensional. This is an explicit advantage of our approach. More details on the approach can be found in Ivanov and Collins (2009).

Note that an (M-mode; $\omega)$ space describes the evolution of a signal in a non-rectangular domain, while (Fourier mode; $\omega)$ can only be applied to a rectangular domain. In other aspects, they are equivalent. It was shown, for example, in Eremeev et al. (1992). Theoretically, we can provide the wave amplitude equation for a quartet in (M-mode, $\omega)$ space. However, Marchesiello et al. (2003), who studied numerically mesoscale variability in the observed area, demonstrated that approximately $30 \%$ of kinetic energy corresponds to nongeostrophic motions. Therefore, we worked only with SSHs, and did not re-calculate them to velocity.

\section{A system of jets, waves and eddies off California}

The mesoscale coherent structures observed in SSH observations off California shared overlapping spatial scales, but had distinct timescales. This allowed for detection and selection of QZJs, BAOs, RWs and MEs. Time-averaged spectra for each of the six frequency bands are shown in Fig. 2a, b as a function of mode number. Figure 2 a shows that the SSH signal was very small for $m>250$ and that QZJs were considerably weaker than annual RWs. Figure $2 b$ indicates that BAOs were weaker than semiannual RWs. Explicit peaks for spectrum $a_{\mathrm{m}}^{2}$ were observed for all frequency bands (Fig. 2a, b) within the domain bounded by the 30th and 250th M-modes. This indicated the existence of energy-dominant scales in the mesoscale flow. The instantaneous spectra were very rough, with multiple narrow peaks (Fig. 2c). The instantaneous spectrum structure was similar to those observed in weakly nonlinear flows (Rabinovich and Trubetskov, 1989).

Characteristic spatial structures for all six frequency bands of the current system are shown in Fig. 3a, b, c, d, e, f. The coherent structures shown in Fig. 3 for the different frequency bands differed from one another and were not stationary. Temporal behavior is discussed below.

QZJs represented eddy chains stretched from east to west, and each chain appeared as a number of eddies of the same sign embedded in an envelope of less intensive zonal shear flow (Fig. 3a). SSH in the jets reached $4-5 \mathrm{~cm}$ and was not less than $2.5-3 \mathrm{~cm}$ within the 17 -year observational 

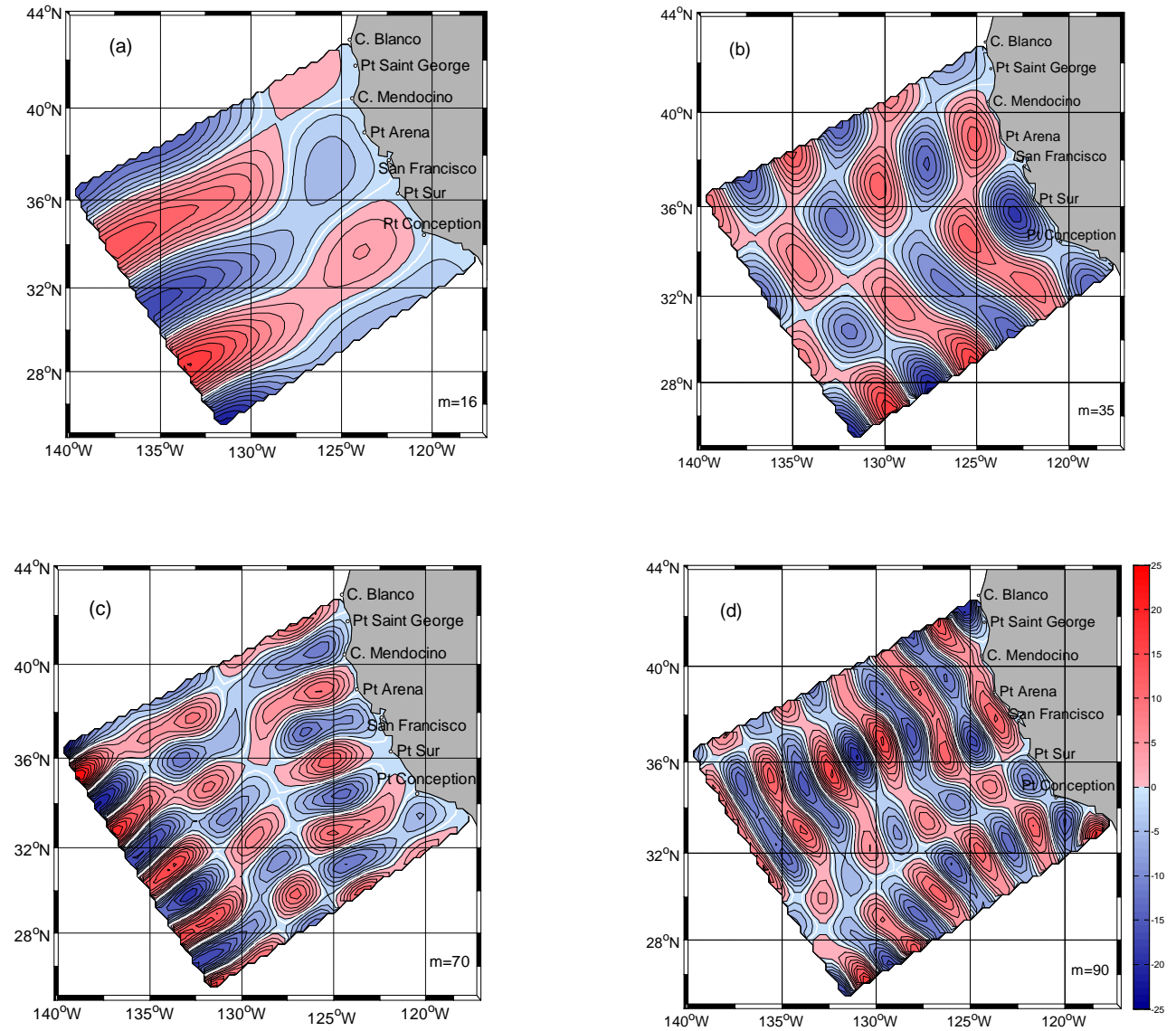

Figure 1. Structure of basis functions (M-modes, $\Psi_{\mathrm{m}} \times 10^{5}$ ) used for calculations. (a) $m=16$, (b) $m=35$, (c) $m=70$, and (d) $m=90$. White contours are zero isolines.

period. The ratio of zonal and meridional scales for the QZJS ( $R=L_{\text {zonal }} / L_{\text {merid }}$ ) varied from $2-3$ (short jets) to 7-8 (long jets). Odd M-modes dominated Eq. (1), which resulted in anisotropy of the QZJ envelope. The direction of the QZJ axes varied from west to southwest. The QZJs also moved towards the Equator at speeds of as great as $0.2 \mathrm{~cm} \mathrm{~s}^{-1}$. However, there was no zonal motion associated with the southward movement. Results clearly indicated that QZJs were regular structures with robust recurrence, and could not be interpreted as statistical fluctuations.

BAOs did not demonstrate any steady motion either to the west or southwest, and their shape differed somewhat from QZJs (Fig. 3b). The structure of BAOs was often close to the structure of standing waves, and therefore they were interpreted as quasi-standing waves. The oscillations were stronger than for QZJs. SSHs for BAOs reached $\sim 5-7 \mathrm{~cm}$.

Propagating RWs with annual and semiannual periodicities were detected and are shown in Fig. 3c, d. Jacobs et al. (1993, 1996) had identified semiannual linear Rossby waves and semiannual oscillations in the Pacific Ocean from satellite data for latitudes as high as 35 and $45^{\circ} \mathrm{N}$, respectively, when barotropic and first baroclinic modes were fit- ted to Geosat data. Ivanov et al. (2012) have analyzed satellite data in the Pacific Ocean within a region from $7^{\circ} \mathrm{N}$ to $60^{\circ} \mathrm{N}$, and found semiannual Rossby waves south of $45^{\circ} \mathrm{N}$. These waves were nonlinear for most of their existence; i.e., they had relatively large steepness (see, for example, Rhines, 2002, 2004).

The annual and semiannual RWs dominated the mesoscale flow off California because SSHs for the waves reached $\sim 10-12 \mathrm{~cm}$. The Rossby waves demonstrated robust recurrence in a sequence of dynamical regimes: linear, amplification and saturation. Figures $3 \mathrm{c}$ and d show the spatial structure for annual and semiannual Rossby waves on 4 October 2006 and 28 November 2007, respectively. The waves propagated westward and southwestward. The propagation speed varied with time, and had maximum values in the linear regime of up to $7-10 \mathrm{~cm} \mathrm{~s}^{-1}$ and a minimum value in the saturation regime when a halt of westward wave propagation was observed. Semiannual waves were more anisotropic than annual waves (compare Fig. 3c to Fig. 3d). Comparing alongshore $\left(L_{h}\right)$ and across-shore $\left(L_{n}\right)$ spatial scales, annual Rossby waves were characterized by $L_{n} \sim L_{h}$ or $L_{n}>L_{h}$. For semiannual Rossby waves, $L_{h} \gg L_{n}$. The structure of 

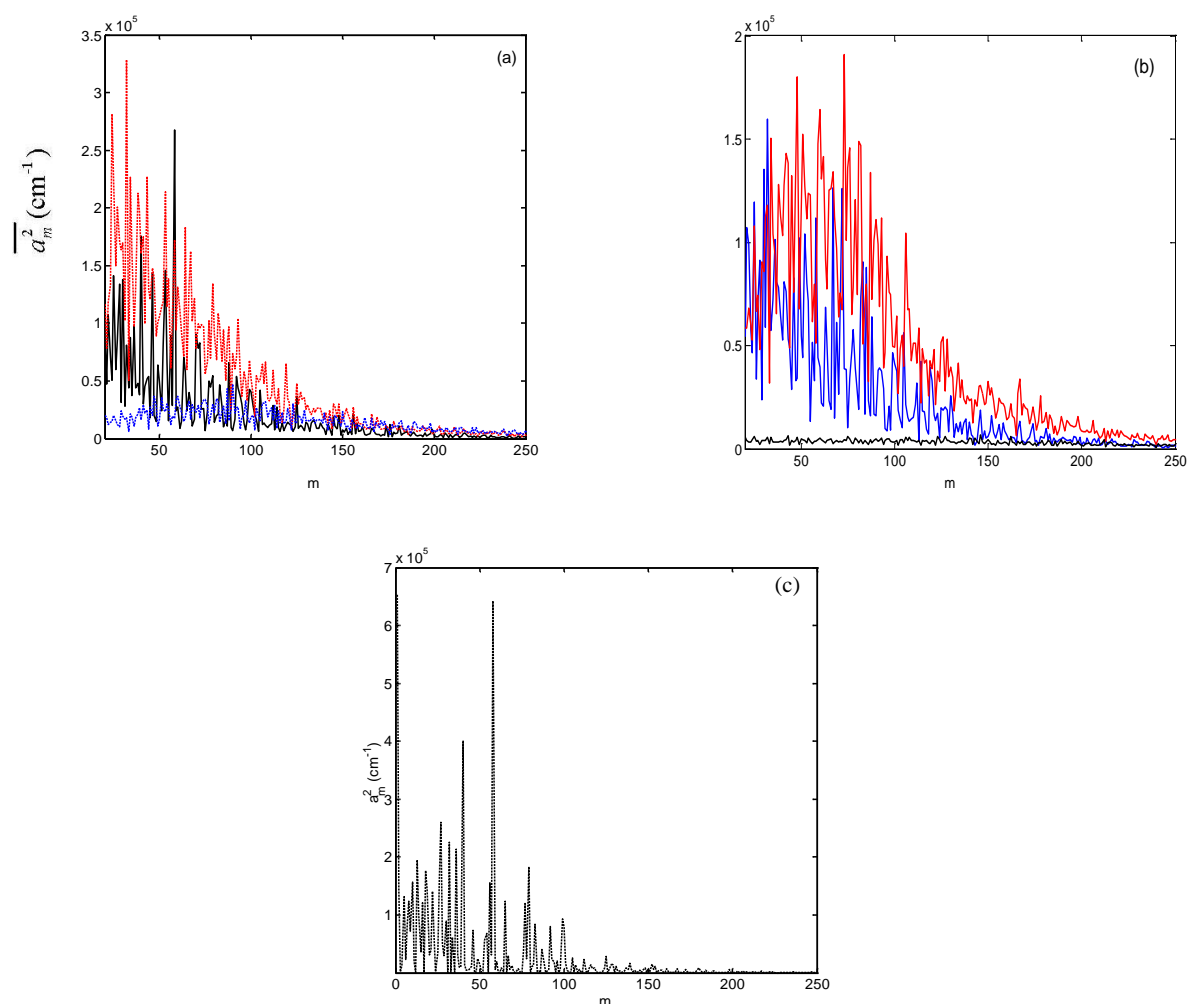

Figure 2. Spectral characteristics of coherent structures observed off California. (a) and (b): time-averaged energy density $\left.\overline{\left(a_{\mathrm{m}}^{2}\right.}\right)$ vs. mode number for the six frequency bands used in this study. (a) Black, red and blue correspond to quasi-zonal jets (with scales longer than 36 months), annual Rossby waves (with scales from 8 to 18 months), and mesoscale eddies (with scales from 2 to 4 months). (b) Blue, red and black correspond to biannual oscillations (with scales from 18 to 36 months), semiannual Rossby waves (with scales from 4 to 8 months), and mesoscale eddies (with scales from 1 to 2 months). (c) An instantaneous spectrum $a_{\mathrm{m}}^{2}$.

semiannual waves was close to meridional. Further discussion of the behavior of the Rossby waves can be found in Ivanov et al. (2010).

MEs-I and MEs-II with scales smaller than RWs were also observed off California (Fig. 3e, f). They formed an eddy field that was statistically inhomogeneous across shore, and demonstrated mean propagation from the coast to the west or southwest. SSH for these eddies reached several $\mathrm{cm}$. To distinguish Rossby waves and mesoscale eddies, the spatial phase coherence criterion (spatial correlation of the phase of the SSH field) was used (Ivanov et al., 2010).

Several features of the coherent structures observed off California were important for the present analysis. First, all structures were generally weakly nonlinear, excluding the cases when $\left\langle\eta^{2}(t)\right\rangle=\iint \eta^{2}(x, y, t) \mathrm{d} x \mathrm{~d} y$ reached maximal and minimal values (Fig. 4). Here, integration was over the computational domain starting on 10 October 1992. Maximum values corresponded to periods when wave steepness $q \sim 2$ (transition to a turbulence regime), and minimal values to $q \ll 1$ (linear structures). Interplay between different M-modes was easily detected via the frequency-phase analysis of M-mode structures in phase space (not shown). The interplay between m-modes will be shown through the analysis of triplets and quartets in the next sections.

Second, the behavior of $\left\langle\eta^{2}(t)\right\rangle$ with time showed robustness for the studied process (Fig. 4). For all six spectral bands, the behavior of this function was robust with time. Here, robustness is a repetition of certain dynamical regimes. Maxima of QZJs corresponded to minima of $\left\langle\eta^{2}\right\rangle$ for BAOs.

Third, a low-frequency modulation (not modulation instability) was visible by eye for all spectral bands shown in Fig. 4 (Rabinovich and Trybetskov, 1993). This modulation became stronger for higher-order spectral bands.

Fourth, all structures observed off California with a horizontal resolution larger than $50-100 \mathrm{~km}$ can be interpreted as weakly nonlinear if $q<1$ and as strongly nonlinear if $q>1$. A transition from weak to strongly nonlinear structures was very quick. However, $q$ was larger than 1 for most of the time (see, for example, Ivanov et al., 2010). We plan to study such transitions and dynamics of strongly nonlinear structures in a separate paper. 

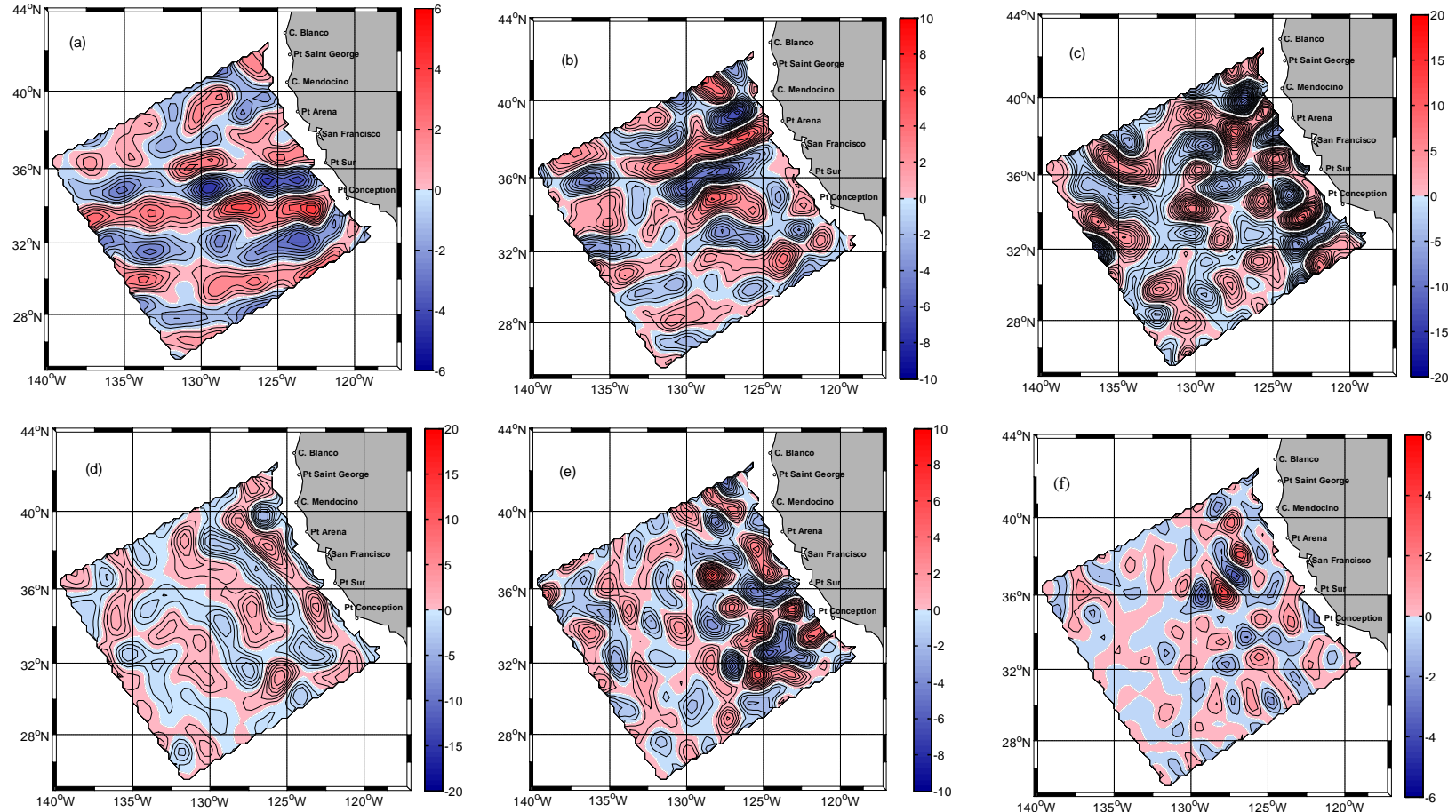

Figure 3. Characteristic spatial structures: (a) quasi-zonal jets (26 May 2006), (b) biannual oscillations (11 January 2006), (c) annual Rossby waves (4 October 2006), and (d) semiannual Rossby waves (28 November 2007). (e) and (f): mesoscale eddies (22 November 2006 and 9 May 2006). Contour intervals are $1 \mathrm{~cm}$ and $0.5 \mathrm{~cm}$ in (a)-(d) and (e) and (f), respectively. Note that the range of the contours varies from \pm 6 in panels (a) and (g) to \pm 10 in (b) and (e) and \pm 20 in (c) and (d).

\section{Wavelet analysis}

RWs and QZJs demonstrated nonlinear behavior. The observed RW steepness was finite (most of the time the steepness was less than or equal to 1) and the QZJs consisted of chains of eddies that stretched from east to west. These eddy chains may indicate the importance of nonlinear dynamics; see, for example, Dritschell and Scott (2011). It is thus logical to study nonlinear mechanisms of QZJ generation and evolution. Numerical and theoretical studies (Gill, 1974; Lee and Smith, 2007, and others) demonstrated that quasi-zonal jets can be generated by resonance as well as near-resonance interactions between flow scales in simplified models.

These interactions should be detected in a phase space that embeds altimetry SSH signals. M-modes were used as a basis to form an appropriate phase space. Next, phase-frequency analysis of M-mode (wave) dynamics was carried out using the amplitude $\left(W_{\mathrm{m}}\right)$ and phase $\left(\phi_{\mathrm{m}}\right)$. Both variables, $W_{\mathrm{m}}$ and $\phi_{\mathrm{m}}$, were determined for each M-mode using the Morlet wavelet transform $\left(\psi_{\tau}\right)$,

$W_{\mathrm{m}}(\tau, t)=\left|W_{\mathrm{m}}(\tau, t)\right| \exp \left\{i \phi_{\mathrm{m}}(\tau, t)\right\}$,

where $\quad W_{\mathrm{m}}(\tau, t)=\int_{-\infty}^{+\infty} a_{\mathrm{m}}\left(t^{\prime}\right) \psi_{\tau}^{*}\left[\left(t-t^{\prime}\right) / \tau\right] \mathrm{d} t^{\prime}, \quad \tau \quad$ is $\quad$ a timescale, and $\omega(m)=\frac{\mathrm{d} \phi_{\mathrm{m}}}{\mathrm{d} t}$ is instantaneous frequency (for details of wavelet techniques, see Kumar and FoufoulaGeorgiou, 1997).

The absolute value of the amplitude $\left|W_{\mathrm{m}}\right|$ describes the evolution of energy-dominant peaks at certain timescales (or frequencies) $\left(\left|W_{\mathrm{m}}\right|^{2}\right.$ is also called the wavelet power spectrum, which, in contrast to the Fourier power spectrum, is a function of time). Given the length of the observational series (nearly 17 years), statistically significant estimates of wavelet amplitudes can be obtained for a domain bounded by 1 month $\leq \tau \leq 6 \mathrm{yr}$ and $8 \mathrm{yr} \leq t \leq 12 \mathrm{yr}$ (Kumar and Foufoula-Gergiou, 1997). Energy-dominant peaks were detected by examining the structure $\left|W_{\mathrm{m}}\right|$. The peaks were selected so as to satisfy one of the following conditions.

Three m-modes participated in near-resonance interactions and formed an appropriate triplet mode if

$m+k=l, \quad|\omega(m)+\omega(k)-\omega(l)| \leq \Omega_{1}$.

Four modes were members of a mode quartet if

$m+k=l, \quad|\omega(m)+\omega(k)-\omega(l)| \leq \Omega_{1}$,

$n-m=l, \quad|\omega(n)-\omega(m)-\omega(l)| \leq \Omega_{2}$.

Here, $\Omega_{1}$ and $\Omega_{2}$ represent resonance broadenings that are caused by finite nonlinearity. Nonlinear interactions are effective if $\Omega_{1}, \Omega_{2} \leq \Gamma_{\mathrm{m}}$, where $\Gamma_{\mathrm{m}}$ is the inverse of the characteristic time of the nonlinear evolution of the mth mode 


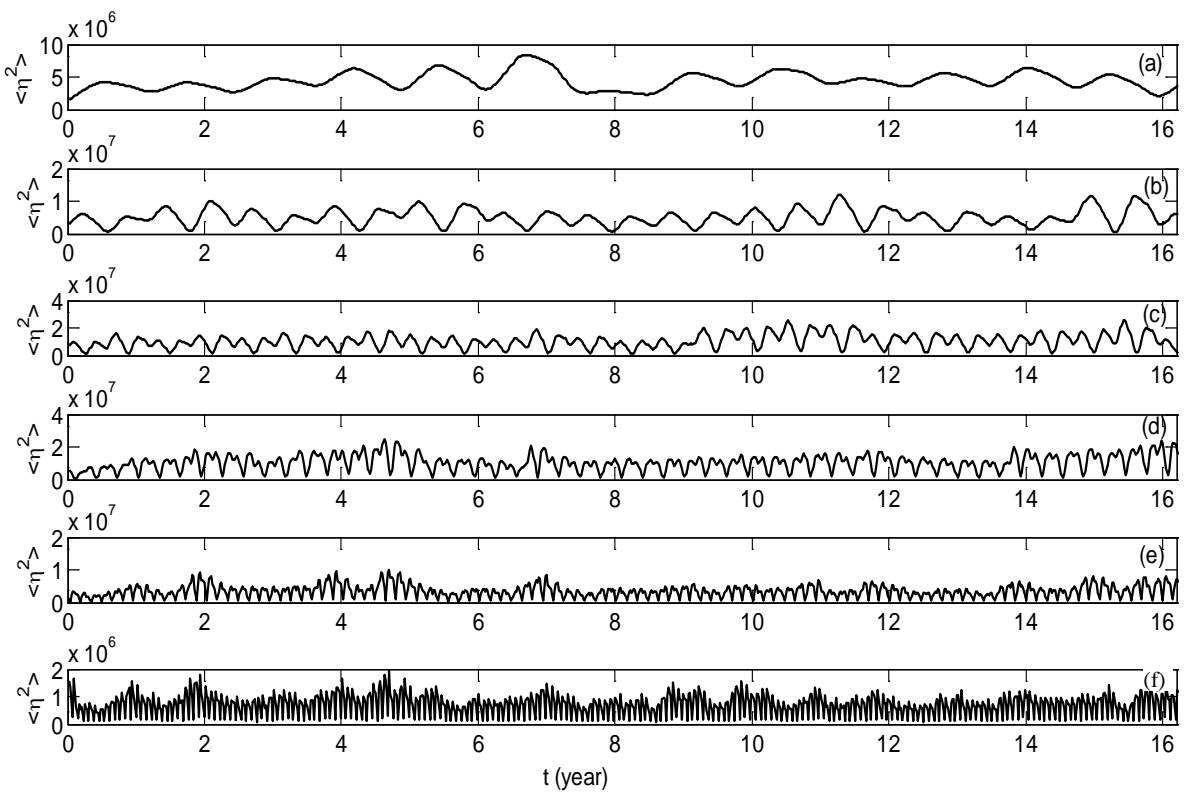

Figure 4. Temporal behavior of $\left\langle\eta^{2}\right\rangle\left(\mathrm{cm}^{2}\right)$ for six spectral bands: (a) longer than 36 months, quasi-zonal jets; (b) 18 to 36 months, biannual oscillations; (c) 8 to 18 months, annual Rossby waves; (d) 4 to 8 months, semiannual Rossby waves; (e) 2 to 4 months, mesoscale eddies; and (f) 1 to 2 months, mesoscale eddies.

(Janseen 2003, Kartashova et al., 2010, and others). For resonance interactions, $\Omega_{1}=\Omega_{2}=0$.

Conditions (5) and (6) are able to describe modulation instability when the lth mode of RW, two mth and nth modes (similar to sideband waves) and the $k$ th mode of lowerfrequency flow interact with one another in a nonlinear sense (Manfredi et al., 2001). Note that conditions (5-6) have to be met during a specific time interval $\delta t$ (this interval was larger than 2 weeks) because either all modes in a quartet fail to exist after nonlinear interactions or all modes continue to exist in quartets, but quartets become unstable and decay due to inhomogeneity of the background flow, vertical temperature gradients, etc.

Since many near-resonance quartets and triplets lived as long as 14 days or longer, as estimated from analysis of the wavelet power spectrum, it was possible to estimate how surface elevation was re-distributed between different scales, and in particular between m-modes for frequency bands corresponding to QZJs, RWs and eddies. Superposition of RW and eddy m-modes (see Eq. 4) results in growth of the QZJ mode, identified from the analysis of the time evolution of all three m-modes (Eq. 4) that are part of a quartet. Quartets with a growth of m-modes (within a mean lifetime) corresponding to QZJs showed that a SSH was re-distributed from high- to low-frequency m-modes. This was a rough estimate, because a part of short-lived m-modes with lifetimes less than 14 days was excluded from our analysis. Conditions 4 6 corresponded to near-resonance nonlinear interactions described by the Poisson bracket $\{\eta, \Delta \eta\}$ for quasi-geostrophic flows or $\{\Psi, \eta\}$ without the geostrophic approximation ( $\Psi$ is "a stream" function) (Pedlosky, 1987). The "stream" function can be defined through a two-scalar potential representation of a three-dimensional incompressible flow; see, for example, Moffatt (1978) or Chu et al. (2003) for applications. Obviously, in this case, the two scalar potentials $\Psi$ and $\Phi$ defined here are not the same as the stream function and velocity potential of a two-dimensional flow (this is discussed in Chu et al., 2003). The statistical stability needed for detection of triads and quartets was achieved through special smoothing with a priori constraints applied (Ivanov et al., 2012a).

Note that horizontal velocity was not estimated from $\mathrm{SSH}$ using geostrophic relationships, because non-geostrophic motions in the studied coastal oceanic area may contain up to $30 \%$ of the total kinetic energy of horizontal circulation (Marchesiello et al., 2003), and frontal dynamics plays an important role in forming circulation in the California Current system (Castelao et al., 2006).

For this study, triads were detected that satisfied (5) as the first step, and then checked to see if two m-modes from this triad formed a second triad (6). If the second triad was (not) found, the existence of quartet (5-6) - triad (4) - was confirmed. Figure 5a, b shows a resonance triad (a) and a nearresonance quartet (b). In both these cases, energy was transferred to low-frequency timescales. The number of possible near-resonance triads and quartets were defined by the choice of resonance broadening.

Our calculations demonstrated the existence of quartets of both resonance and non-resonance nature, and resonance and non-resonance triplets at the same time. 

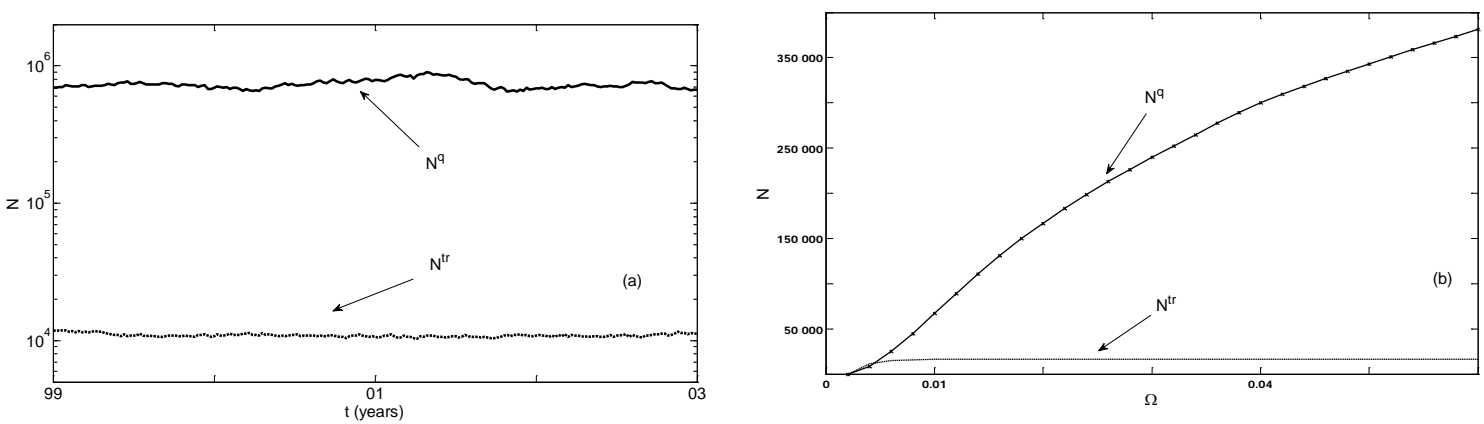

Figure 5. Variability of the number of near-resonance triplets, $N^{\mathrm{tr}}$, and near-resonance quartets, $N^{\mathrm{q}}$, as a function of (a) time and (b) resonance broadening, $\Omega$.

Note that, to understand energy re-distribution within the energy spectrum, the energy transfer integrals need to be calculated; for example, see Sagaut and Cambon (2008). Unfortunately that cannot be done, because equations describing SSH anomaly motions contain terms that cannot be determined from satellite altimetry data only. Therefore, simpler characteristics were calculated that described the re-distribution of the altimetry signal between scales (see Sect. 6). This approach allowed calculation of general characteristics of the re-distribution of energy. Generally, "inverse" transfer from fast motions to slower motions was observed, but not from small to large spatial scales.

Triplet and quartet interactions showed different mechanisms of energy re-distribution between scales, as well as different timescales for generation of QZJs. For example, Loesch (1977) demonstrated that the timescale required for jet generation by quartet interactions was an order of magnitude longer than that associated with triad interactions.

\section{Resonance and near-resonance interactions}

On average, between 1999 and 2003, at each moment in time, $\sim 40-80$ resonance triads $\left(\Omega_{1}=0\right), \sim 3-5$ resonance quartets $\left(\Omega_{1}=0, \Omega_{2}=0\right)$, about $2 \times 10^{4}$ near-resonance triads $\left(0<\Omega_{1}<\Omega^{*}\right)$ and $1.75 \times 10^{5}$ near-resonance quartets $(0<$ $\Omega_{1}<\Omega^{*}, 0<\Omega_{2}<\Omega^{* *}$ ) were detected (see Fig. 6). For simplicity, hereafter, $\Omega^{*}<\Omega^{* *}=0.01$ radians. The number of triads and quartets changed with time, but not very quickly (Fig. 5a). The number of quartets decreased as $\Omega^{*} \sim \Omega^{* *} \rightarrow 0$, but was always larger than the number of triads if $\Omega^{*}>\Omega_{\mathrm{cr}}$ for reasonable values of resonance broadening (Fig. 5b).

The stability of resonance quartets and triads depends on the inhomogeneity of the background conditions of the ocean (density gradient, shear flow, etc). This inhomogeneity should destroy exact resonances, and most likely exact resonances dominate only in simplified theoretical models. Therefore, in mesoscale oceanic flows, near-resonance interactions are more likely. The intensity of these interactions is determined by the resonance broadening and kinematic features of near resonances.

Squared amplitudes for most quartets were also larger (some amplitudes were much larger) than the squared amplitudes of triads (not shown). The dominance of near-resonant quartets compared to resonant and near-resonant triads indicated that there was modulation instability of Rossby waves off California: modulations of the Rossby wave envelope excited zonal flows through a nonlinear mechanism (probably Reynolds stress).

The number of near-resonance quartets $\left(N^{\mathrm{q}}\right)$ and the number of near-resonance triads $\left(N^{\text {tr }}\right)$ did not change much with time (Fig. 5a). Quartets dominated off central California. $N^{\mathrm{q}}$ was much larger than $N^{\mathrm{tr}}$, except for values of $\Omega \ll 0.01$ radians (Fig. 5b). Squared amplitudes, $a_{\mathrm{m}}^{2}$, for most quartets were also larger (some amplitudes were much larger) than the squared amplitudes of triads (not shown).

\section{Re-distribution of surface elevation between scales}

Kinematic analysis was applied to understand how mean squared SSH was re-distributed between different scales. The number of quartets for which a signal was transferred from waves and eddies to QZJs and BAOs is shown in Fig. 6a. (Note that this number was considerably less than the total number of existing quartets shown in Fig. 5a.) This indicated a dominant role for four-wave interactions (modulational instability) in the process of forming SSH spectra as compared to three-wave interactions.

M-modes for QZJs and BAOs had mean timescales of $4.3 \pm 0.7$ years and $2.4 \pm 0.9$ years, respectively (Fig. 6b). Variations of both mean timescales were not large, which reflected a high degree of regularity of QZJs and BAOs off California.

Since the wave steepness, $q$, can be larger than 1 for quite short time periods associated with a transition to turbulence, under some conditions, an inverse cascade can occur or coexist with modulational instability. Naturally, when $q \ll 1$, pure modulational instability dominates (Connaughton et al., 

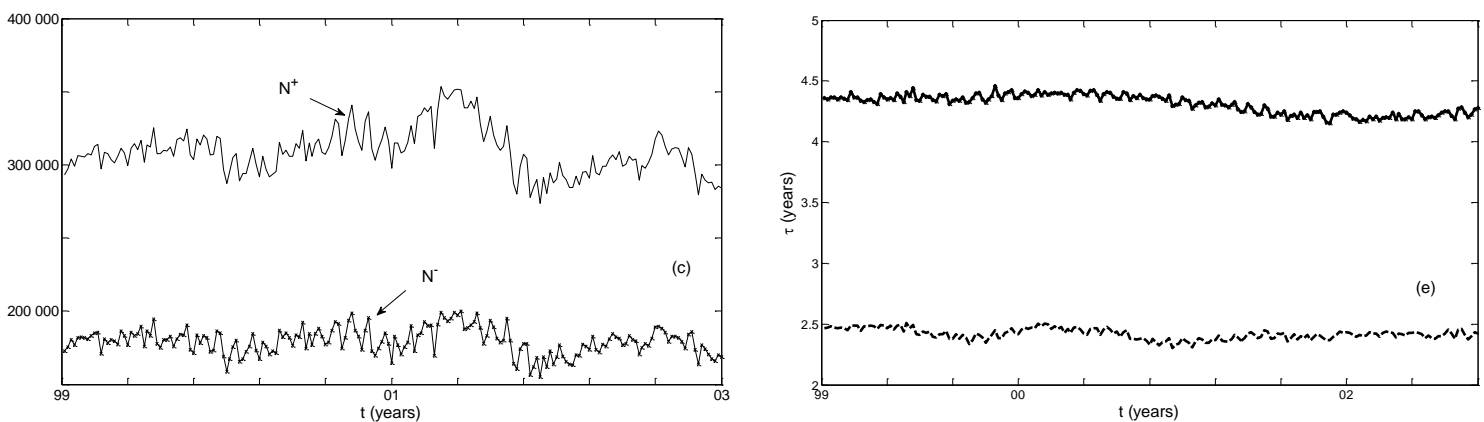

Figure 6. Temporal behavior of the number and timescale of near-resonance quartets corresponding to quasi-zonal jets and biannual oscillations in 1999-2003. (a) Number of near-resonance quartets that participated in inter-scale interactions to generate the energy for quasi-zonal jets (QZJs) $\left(N^{+}\right)$and biannual oscillations (BAOs) $\left(N^{-}\right)$. (b) Mean timescales for QZJs $(-)$and BAOs $(-)$.

2010). It is therefore important to look for the existence of an inverse cascade in our analyses.

Two criteria were used to determine and identify whether or not an inverse cascade formed QZJs. First, spectral entropy was used to estimate the re-distribution of SSH signals between different m-modes (Aubry et al., 1991). The spectral entropy, $S$, was calculated as

$S(M, t)=-(\log M)^{-1} \sum_{m=m_{o}}^{M} p_{\mathrm{m}} \log p_{\mathrm{m}}$,

where $p_{\mathrm{m}}(t)=a_{\mathrm{m}}^{2} / a(t) ; a(t)=\sum_{m=m_{o}}^{M} a_{\mathrm{m}}^{2}(t), \mathrm{S}$ equals one for a uniform distribution of a signal among all $\left(M-m_{0}\right)$ modes, and equals zero when the signal was contained in a single mode.

Figure 7a shows the behavior of $S(M, t)$ with time for modes numbered from $m_{0}=30$ to $M=250$. This figure shows that there was no explicit signal flux from small scales to large scales of SSH during the entire observational period. $S(M, t)$ fluctuated from 0.68 to 0.72 . Increasing $M$ to 1000 increased the mean value of $S$ only slightly, to 0.72 . These results indicated that either there was no inverse cascade of energy or that the cascade was weak.

The centroid for the spectrum of $\eta^{2}$ from $m_{0}=30$ to $M=$ 1000 was also calculated to identify inverse cascade. Figure $7 \mathrm{~b}$ demonstrates the behavior of the spectral centroid with time. A simple analysis of this behavior is that there were time intervals within which the centroid shifted to larger scales. To illustrate the character of this shift, the spectrum of $a_{\mathrm{m}}^{2}$ for day 2940 (point A in Fig. 8a) when the centroid was 82 (Fig. 8 b) was contrasted with the spectrum on day 3150 (point B in Fig. 8a), when the centroid had decreased to 62 (Fig. 8c). The spectra after the shift (Fig. 8c) showed a decay of energy for M-modes with $m>100$. From another perspective, $\eta_{m}^{2}$ did not demonstrate a power behavior with $m$ (not shown). This indicated that the classical inverse cascade was not observed in our analysis of the satellite data. A specific mechanism for the reduction of the spectral centroid
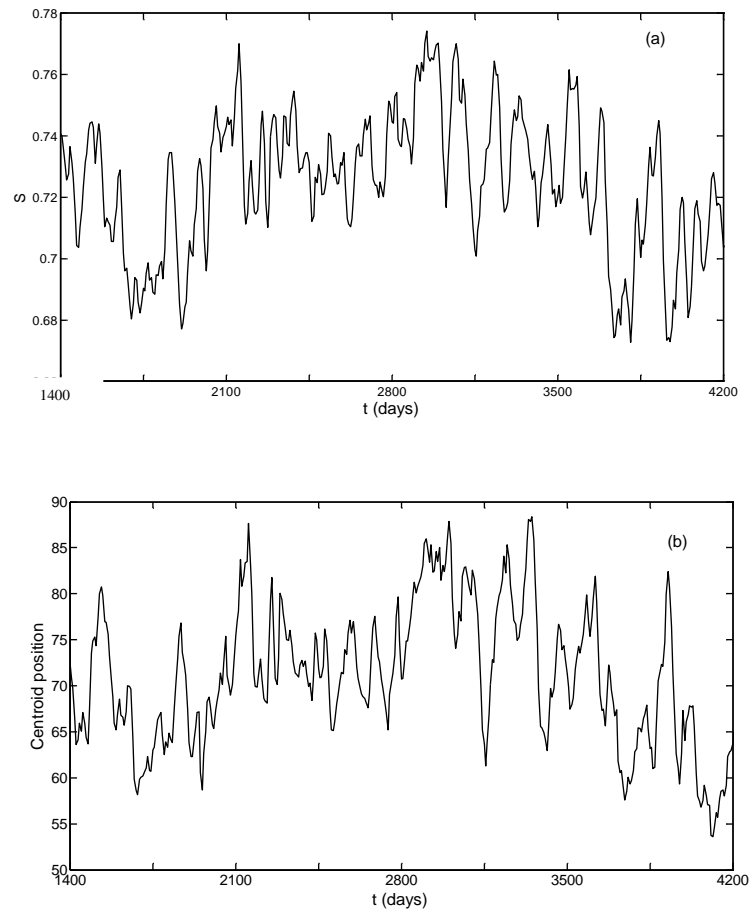

Figure 7. Evolution of spectral entropy and centroid, 10 October 1992 to 23 May 2009. (a) Spectral entropy in non-dimensional units. (b) Spectral centroid in $\mathrm{m}$.

position will be studied in another paper. It may be caused by three-dimensional or other effects.

The mechanism of QZJ generation due to near-resonance inter-scale interactions is similar to that suggested by Lorenz (1972) for barotrophic flow, and was originally called "Rossby wave instability" by Lorenz. A finite-amplitude Rossby wave interacts with a zonal flow perturbation on a $\beta$ plane. The surfaces of a constant phase move to the west. The zonal flow tends to distort them. At this point, it is easy to see that the waves being transverse, fluid particles, whose velocity is parallel to the wave front, need to turn to the west. This 

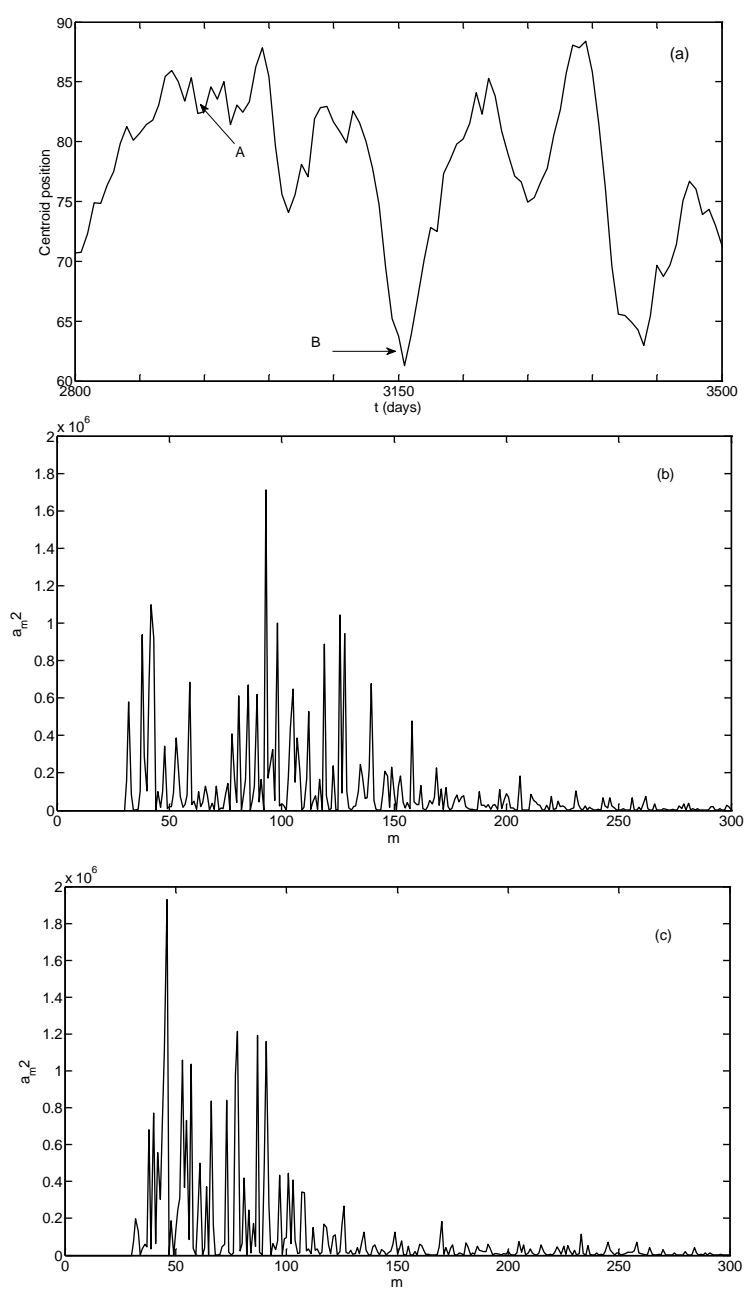

Figure 8. An evolution of the spectral centroid. (a) Spectral centroid from 5 August 1999 to 5 July 2000. A and B indicate a time interval within which the centroid moved to the lowest value. (b) Spectrum of $a_{\mathrm{m}}^{2}$ for point A on day 2940. (c) Spectrum of $a_{m}^{2}$ for point B on day 3150 .

imparts eastward momentum when the fluid particles cross the zonal flow perturbation. This continuous deposition of eastward momentum by the wave produces a positive feedback mechanism leading to exponential growth of the zonal perturbation.

However, in contrast to Lorenz (1972), modulational instability dominated the mesoscale flow off California, and there was channeling of energy not only to QZJs, but also to BAOs. This is a typical self-organized process. Energy was pumped by wind into the ocean and then it was re-distributed between scales, so that robust regular structures (QZJs and BAOs) were formed due to nonlinear interactions between scales (Nicolis and Prigogine, 1977).

Note that the oceanic eastern boundary current off California is a baroclinic system. SSHs observed from satellites are also a baroclinic system. The baroclinicity can influence the modulational instability in two ways. First, if waves participating in modulation instability seem to be barocinicly stable, then their wave characteristic scales and others should be different than for barotropic waves only. Second, for Rossby waves generated by baroclinic instability, a small modulation with a period of the same order as the internal dynamical timescale can completely destabilize the nonlinear system, leading to chaotic solutions even for small supercriticality; see, for example, Hart (1979, 1989) and others.

\section{Role of flow dissipativity}

Lee and Smith (2007) suggested that the resonance broadening $\Omega=\gamma \cdot R n$, where $\gamma$ is a small parameter and $R n$ is a non-dimensional Rhines number, the latter defined for the $\beta$ plane system as the ratio of linear and nonlinear timescales. With a characteristic length scale $L$ and a nonlinear timescale $L / U$, the Rhines number is equal to $U /\left(L^{2} \beta\right)$. The resonance broadening seen above is determined by the dissipativity of mesoscale flow caused by bottom friction, vertical viscosity, etc. The Rhines scale depends on the level of dissipativity and the inhomogeneity of the background medium (density gradient, shear flow, etc.; Danilov and Gurarie, 2002). This inhomogeneity should destroy exact resonances, and exact resonances probably dominate only for simplified theoretical models. Near-resonance interactions seem more likely for mesoscale oceanic flows. Intensity of near-resonance interactions is determined by resonance broadening and kinematic features of near resonances.

Therefore, dissipativity of mesoscale flow should play an important role in the re-distribution of energy between scales, because its level determines the number of near resonances involved in energy exchange between scales. This can be illustrated by assuming that linear bottom friction with coefficient $\lambda$ dominated the mesoscale flow. The Rhines number is defined as

$R n \sim \beta^{-1} \lambda^{-1 / 2} \varepsilon^{1 / 2} L^{-1}$,

where $\beta$ and $\varepsilon$ are gradients of the local Coriolis parameter and injection rate, respectively. Equation (8) is similar to that given in Danilov and Gurarie (2002) for a two-dimensional flow, although it can also be applied in the case of threedimensional flows. This is true if the injection rate $\varepsilon$ in Eq. (8) is modified to include the contribution of open boundary conditions (if any). Since $\varepsilon$ was not determined for the CCS, the injection rate in Eq. (8) was not changed.

Nonlinear interactions are effective if $\Omega<\Gamma_{\mathrm{m}}$ (Rabinovich and Trubetskov, 1989). For simplicity, we replace $\Gamma \sim \Gamma_{\mathrm{m}}$, and with $\Omega=\gamma R n$, then $\lambda$ in the flow should be limited as

$\lambda>\gamma^{2} \Gamma^{-2}(\beta / 2)^{-2} \varepsilon L^{-2}$.

Since the value of the injection rate is not known (at least for the California Current system), this demonstrates only that 
there exists a threshold for $\Omega$. Below this threshold, when the number of near-resonance quartets is strongly reduced as $\lambda$ tends to zero, the energy transfer into QZJs should cease. The same conclusion can be made for BAOs as well.

So, QZJs require the existence of some finite level of dissipativity. If this level is not reached, no zonal jets can be found in a flow. This conclusion agrees with results obtained by Berloff et al. (2011), who also found that jet generation requires the existence of some finite level of dissipation in a system. Another issue is how the choice of dissipativity controls the latency of QZJs.

Chelton et al. (2011) have hypothesized that propagating SSH anomalies off California was a response of the ocean surface to mesoscale eddies, because there is "practically" no wave dispersion for such scales. Estimates obtained in this study and in Ivanov et al. (2010) have shown that wave dispersion is finite. Rossby waves of annual and semiannual periodicities that participate in quartets are clearly dispersion waves at least in linear and quasi-linear regimes of propagation (Ivanov et al., 2010). They also participate in modulational instability. Therefore, these SSH anomalies should be interpreted as a result of forcing by nonlinear Rossby waves.

Note that bottom friction is very important for generation of broadening of the spectral peaks that correspond to waves. The point is that any turbulent process generates broadening of the peaks, and turbulence is always present in oceanic flows. The broadening of the peaks by turbulence is described in the Appendix of Galperin et al. (2010).

\section{Phase synchronization/locking events}

A number of mechanisms form QZJs in the ocean. QZJs can be generated by near-resonance triple interactions between modes (e.g., see Lee and Smith, 2007), anomalous local wind forcing, as assumed in Nakano and Hasumi (2005), or by the instability of background currents (e.g., Berloff et al., 2009). However, in each of these cases, if nonlinear interactions including quartets play an important role, phase synchronization/locking events should be expected. These can easily be extracted from observations in phase space (Pikovsky et al., 2001).

The M-modes introduced by Eq. (1) generate an appropriate phase space. Hence, the underlying structure of quasizonal jets and inter-scale interactions forming these jets can be analyzed through the phase-frequency analysis of $\mathrm{M}$ mode dynamics using amplitude-phase variables. Phase synchronization corresponds to the case when the phase difference between two oscillations is zero. Phase locking assumes that this difference is constant (Pikovsky et al., 2001).

Using (2) does not solve the problem of phase synchronization/locking events, because estimates have shown that in this case, only the 1999-2001 phases can be observed. Therefore, another definition of phase synchronization/locking events based on Pereira et al. (2007) is used.
They suggested that phase synchronization/locking events in a nonlinear system can be detected through correlations between local maxima and minima of system variables in phase space. In this study, amplitudes of M-modes were used as system variables. Two modes were in phase (anti-phase) if the difference between two times $t_{j}$ and $t_{j *}$ corresponding to two neighboring maxima or two neighboring minima was less than some threshold $\delta$; i.e.,

$\left|t_{j}-t_{j *}\right| \leq \delta$

$\delta=0$ corresponds to phase synchronization, and $\delta \neq 0$ is phase locking. Note that, in comparison with traditional techniques for calculation of the phase of a multi-frequency signal as a wavelet or Hilbert transform, the above approach is applicable to any signal that contains distinct marker events.

Appropriate histograms calculated as discussed above are shown in Fig. 9. It is clear from these histograms that quartets (four-wave interacting structures) were detected in two different spectral bands. First, modes corresponding to QZJs, annual Rossby waves, semiannual Rossby waves and MEs from the fifth spectral band formed quartets that were responsible for energy transfer from Rossby waves to the QZJS (Fig. 9a, c, e, g). Phases of all these modes were locked, which indicates strong interactions between these modes. These quartets corresponded to maximum values of $\eta^{2}$. The quasi-zonal jets had maximum lengths (long jets) when the number of synchronization events was at a maximum.

Second, modes that corresponded to BAOs (with scales from 2 to 3 years) correlated with semiannual Rossby waves and mesoscale eddies from the fifth and sixth spectral bands (Fig. 9b, d, f, h). Phases of all these modes were locked, which indicates strong interactions between these modes. In this case, the energy was transferred from Rossby waves to BAOs. Forming BAOs from semiannual Rossby waves demonstrated the possibility of inverse cascades of energy to accelerate this process. Again, these quartets corresponded to maximum values of $\eta^{2}$. However, since BAOs are not QZJs, they cannot have a maximum length when the number of synchronization/locking events is at a maximum, but modes forming these oscillations have maximum amplitudes too.

Note that QZJS and BAOs did not interact with one another over the length of the data record. However, this does not mean that these processes were linear.

\section{Conclusions}

Some evidence of a self-organized process in the CCS is given here. It occurred when QZJs and BAOs were formed due to near-resonance inter-scale interactions through modulational instability. The number of observed quartets was 10 times larger than the number of induced triads. As a consequence, the SSH signal was transferred from annual and semiannual Rossby waves and mesoscale eddies to lowerfrequency current structures such as QZJs and BAOs. This 

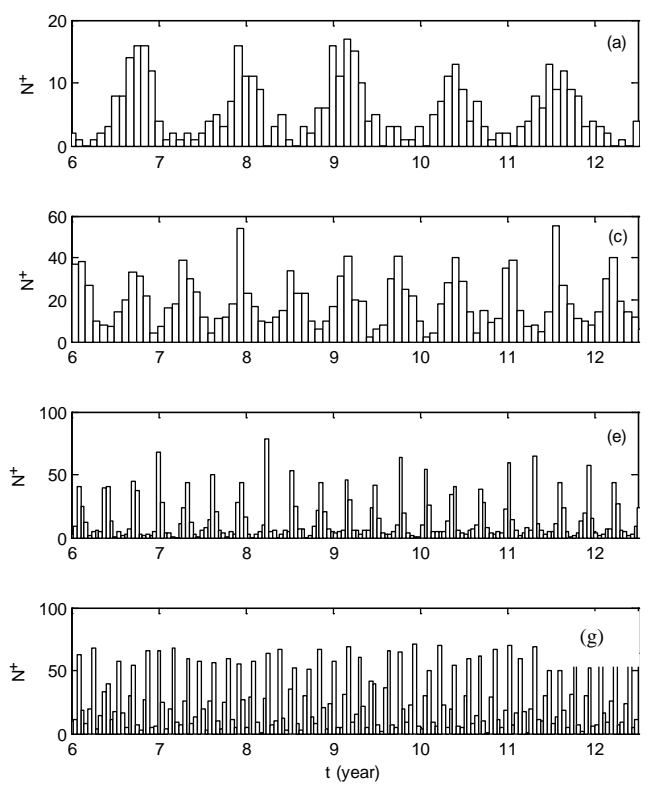
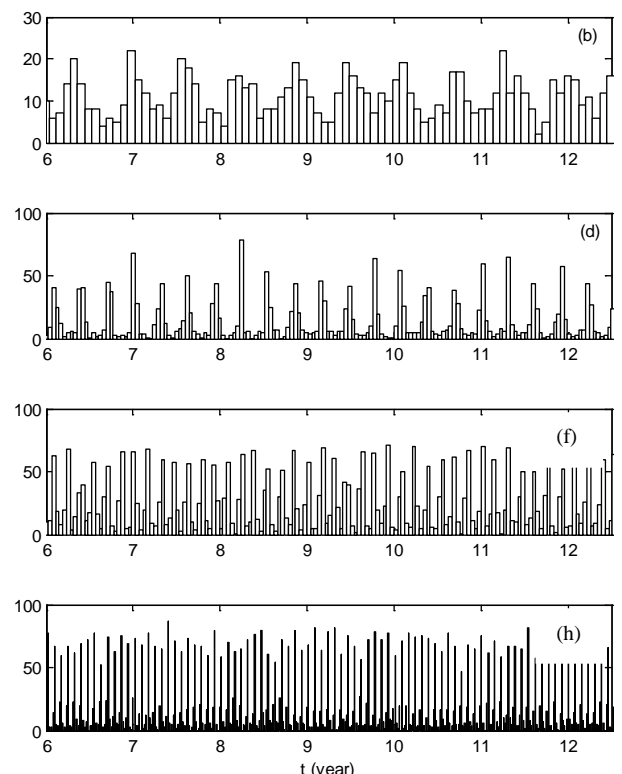

Figure 9. Synchronization/locking events off California. (a, c, e, h) The first quartets among the quasi-zonal jets, annual Rossby waves, semiannual Rossby (SARWs) waves and mesoscale eddies from the fifth band. (b, d, $\mathbf{g}, \mathbf{f}$ ) The second quartets formed by biannual oscillations, SARWs and mesoscale eddies from the fifth and six spectral bands. $N^{+}$is a number of synchronized modes.

example demonstrates modulational (Benjamin-Feir) instability in which the carrier wave is phase locked with the side bands.

Two types of quartets were observed off California. Quartets of the first type contained QZJs, annual Rossby waves, semiannual Rossby waves and mesoscale eddies from the fifth spectral band. Note that the mesoscale eddy field was strongly smoothed by the AVISO team. Therefore, although the harmonics that participated in nonlinear interactions were identified more or less accurately, fluxes between harmonics have been shifted into the Rossby wave band.

The second type of quartet consisted of BAOs, semiannual Rossby waves and mesoscale eddies from the fifth and sixth bands. Energy transfer to BAOs from faster currents seems to be a novel phenomenon, and has not been noted in the modern oceanographic literature (Baldwin et al., 2001).

Note that it was found that BAOs did not interact with QZJs nonlinearly within the given observational series. However, this does not mean that BAOs or QZJs should be approximately linear, although they are the lowest frequency currents observed in satellite data.

Dissipativity of an oceanic flow controls generation of QZJs and BAOs. This is because the level of dissipativity determined the number of near resonances, causing non-local energy transfer from fast to slower timescales (inverse time cascade). A minimum dissipativity level in the CCS was shown to exist (it was demonstrated, for example, for bottom friction) when QZJs and BAOs were generated.
These results help in understanding the role of QZJs in the CCS. Although the QZJs have a considerably weaker signature in SSH anomalies than RWs, they acted as a catalyst, helping RWs, MEs and QZJs exchange energy between themselves. In our opinion, QZJs could not serve as barriers for meridional transport in the CCS, because they are too weak.

Conclusions made in this paper apply only to the California Current system. Other physical mechanisms for generation of QZJs may exist in other regions. For example, Tanaka and Akitono (2010) found the existence of two different mechanisms in a two-layer model of an idealized ocean. Note that our analysis does not support Centurioni et al. (2008), who discussed direct instability of the CCS meanders and hypothesized a possible mechanism of QZJ generation in this region as plumes.

Acknowledgements. Support for LI (CC) was provided by NSF grant OCE-0827527 (OCE-0827160). The authors thank J. McWilliams (UCLA), E. Pelinovsky (Institute of Applied Physics, Russia) and B. Galperin (University of South Florida) for useful discussions of these results.

Edited by: R. Grimshaw

Reviewed by: two anonymous referees 


\section{References}

Aubry, N., Guyonett, R., and Lima R.: Spatio-temporal analysis of complex signals: theory and applications, J. Stat. Phys., 64, 683739, 1991.

Baldwin, M. P., Gray, L. G., Dunkerton, T. J., Hamilton, K., Haynes, P. H., Randel, W. J., Holton, J. R., Alexander, M. J., Hirota, I., Horinouchi, T., Jones, D. B. A., Kinnersley, J. S., Marquardt, C., Sao, K., and Takahasi, M.: The Quasi-Biennial Oscillation, Rev. Geophys., 39, 179-229, 2001.

Baldwin, M. P., Rhines, P., Huang, H.-P., and McIntyre, M.: The jet-stream conundrum, Science, 315, 467-468, 2007.

Benjamin, T. B. and Feir, J. E.: The disintegration of wave trains on deep water, J. Fluid. Mech., 27, 417-430, 1967.

Berloff, P., Kamenkovich, I., and Pedlosky, J.: A mechanism of formation of multiple zonal jets in the oceans, J. Fluid Mech., 628, 395-425, 2009.

Berloff, P., Karabasov, S., Farrar, T., and Kamenkovich, I.: On latency of multiple zonal jets in the oceans, J. Fluid. Mech., 686, 534-567, 2011

Castelao, R. M., Mavor, T. P., Barth, J. A., and Breaker, L. C.: Sea surface temperature fronts in the California Current System from geostationary satellite observations, J. Geophys. Res., 111, C09026, doi:10.1029/2006JC003541, 2006.

Centurioni, L. R., Ohlman, J. C., and Niiler, P. P.: Permanent meanders in the California Current System, J. Phys. Oceanogr., 38, 1690-1710, 2008.

Chelton, D. B., Schlax, M., and Samelson, R. M.: Global observations of nonlinear mesoscale eddies, Prog. Oceanogr., 91, 167216, 2011.

Chu, P. C., Ivanov, L. M., Korzhova, T. P., Margolina, T. M., and Melnichenko, O. V.: Analysis of sparse and noisy ocean current data using flow decomposition, Part 1, J. Atmos. Ocean Tech., 20, 478-491, 2003.

Collecte Localisation Satellites, SSALTO/DUACS user handbook: (M) SLA and (M) ADT near-real time and delayed time products, CLS-DOS-NT-06.034, Ramonville Saint-Agne, France, 54 pp., 2006.

Connaugton, C., Nadiga, B., Nazarenko, S., and Quinn, B.: Modulational instability of Rossby and drift waves and generation of zonal jets, J. Fluid Mech., 654, 207-231, 2010.

Danilov, S. and Gurarie, G.: Rhines scale and spectra of the $\beta$ plane turbulence with bottom drag, Phys. Rev. E, 65, 0673011-067301-3, 2002.

Dritschell, D. G. and Scott, R. K.: Jet sharpening by turbulent mixing, Philos. Tran. A, 369, 754-778, 2011.

Eremeev, V. N., Ivanov, L. M., and Kirwan Jr, A. D.: Reconstruction of oceanic flow characteristics from quasi-Lagrangian data: 1. Approach and mathematical methods, J. Geophys. Res., 97, 2156-2202, doi:10.1029/92JC00356, 1992.

Galperin, B., Sukoriansky, S., and Dikovskaya N.: Geophysical flows with anisotropic turbulence and dispersive waves: flows with a beta-effect, Ocean. Dynam., 60, 427-441, 2010.

Gill, A. E.: The stability of planetary waves on an infinitie betaplane, Geophys. Fluid Dynam., 6, 29-47, 1974.

Hart, J. E.: Finite amplitude baroclinic instability, Ann. Rev. Fluid. Mech., 11, 147-172, 1979.

Hart, J. E.: Finite-amplitude baroclinic instability with periodic forcing, Physica D, 39, 239-261, doi:10.1016//01672789(89)90007-9, 1989.
Hristova, H., Pedlosky, J., and Spall, M. A.: Radiating instability of meridional boundary current, J. Phys. Ocean., 38, 2294-2307, 2008.

Ivanov, L. M. and Collins, C. A.: Modal decomposition of oceanic circulation: Applications for high-resolution models and Lagrangian data, in: Chapter 2, Ocean Circulation and El Niño, New Research Nova Science Publishers, Inc., New York, 31-69, 2009.

Ivanov, L. M., Collins, C. A., and Margolina, T. M.: System of quasi-zonal jets off California revealed from satellite altimetry, Geophys. Res. Lett., 36, L03609, doi:10.1029/2008GL036327, 2009.

Ivanov, L. M., Collins, C. A., Margolina, T. M., and Eremeev, V. N.: Nonlinear Rossby waves off California. Geophys. Res. Lett., 37, L13602, doi:10.1029/2010GL043708, 2010.

Ivanov, L. M., Collins, C. A., and Margolina, T. M.: Jets, Rossby Waves and Eddies off California, in Proc.: 20 Years of Progress in Radar Altimery, Venice, 46-52, 2012.

Jacobs, G. A., Emery, W. J., and Born, G. H.: Rossby waves in the Pacific Ocean extracted from Geosat altimetry data, J. Phys. Oceanogr., 1155-1175, 1993.

Jacobs, G. A., Teaque, W. J., Mitchell, J. L., and Hurlburd, H. E.: An examination of the North Pacific Ocean in the spectral domain using Geosat altimeter data and a numerical ocean model, J. Geophys. Res., 101, 1025-1044, 1996.

Janseen, P. A.: Nonlinear four-wave interactions and Freak waves, J. Phys. Oceanogr., 33, 863-884, 2003.

Kartashova, E., Lvov, V., Nazarenko, S., and Procaccia, I.: Towards a Theory of Discrete and Mesoscopic Wave Turbulence, Technical report no. 10-04 in RISC Report Series, Research Institute for Symbolic Computation, 2010.

Kumar, P. and Foufoula-Georgiou, E.: Wavelet analysis for geophysical applications, Rev. Geophys., 34, 385-412, 1997.

Lee, Y. and Smith, L. S.: On the formation of geophysical and planetary zonal flows by near-resonance wave interactions, J. Fluid Mech., 576, 405-424, 2007.

Loesch, A. Z.: On generation of zonal flows by interacting Rossby waves, Tellus, 29, 306-316, 1977.

Lorenz, E. N.: Barotrophic instability of Rossby wave motion, J. Atmos. Sci., 29, 258-264, 2007.

Manfredi, G., Roach, C. M., and Dendy, R. O.: Zonal flow and streamer generation in drift turbulence, Plasma Phys. Control. Fusion, 43, 825-835, 2001.

Marchesiello, P., McWilliams, J. C., and Shchepetkin, A.: Equilibrium structure and dynamics of the California Current System, J. Phys. Oceanogr., 33, 753-783, 2003.

McWilliams, J. C.: Fundamentals of Geophysical Fluids Dynamics, Cambridge University Press, Cambridge, 2006.

Moffatt, H. K.: Magnetic Field Generation in Electrically Conducting Fluids, Cambridge University Press, 578 pp., 1978.

Nadiga, B.: On zonal jets in oceans, Geophys. Res. Lett., 33, L10601, doi:10.1029/2006GL025865, 2006.

Nakano, H. and Hasumi, H.: A series of zonal jets embedded in broad zonal flows in the Pacific obtained in eddy-permitting ocean general circulation models, J. Phys. Oceanogr., 35, 474488, 2005.

Newell, A.: Rossby wave packet interactions, J. Fluid. Mech., 35, 255-271, 1969. 
Nicolis, G. and Prigogine, I.: Self-Organization in Non-Equilibrium Systems, Wiley, New York, 1977.

Pedlosky, J.: Geophysical Fluid Dynamics, 2nd Edn., Springer, New York, 625 pp., 2007.

Onishenko, O. G., Pokhotelov, O. A., and Astafieva, N. M.: Generation of large scale eddies and zonal winds in planetary atmosphere, Physics Uspekhi, 51, 577-589, 2008.

Pereira, T., Baptista, M. S., and Kurths, J.: Detecting phase synchronization by locates maps: Application to neural networks, Eur. Phys. Lett., 77, 40006, doi:10.1209/0295-5075/77/40006, 2007.

Pikovsky, A., Rosenblum, M., and Kurths, J.: Synchronization: A Universal Concept of Nonlinear Sciences, Cambridge Press, 2001.

Qiu, B., Chen, S., and Sasaki H.: Generation of the North Equatorial undercurrent jets by triad baroclinic Rossby wave interactions, J. Phys. Oceanogr., 43, 2682-2698, 2013.

Rabinovich, M. I. and Trubetskov, D. I.: Oscillations and Waves: in Linear and Nonlinear Systems, Springer Verlag, Berlin, 1989.

Rhines, P. B.: Wave s and turbulence on beta-plane, J. Fluid Mech., 69, 417-443, 1975.

Rhines, P. B.: Rossby Waves, Academic Press, Salt Lake City, 1-37, 2002.
Rhines, P. B.: Oceanic and Atmospheric Rossby Waves, Norman Phillips Symposium at the Annual meeting of the American Meteorological Society, Boston, 41 pp., 2004.

Sagaut, P. and Cambon, C.: Homogenous Turbulence Dynamics, Cambridge University Press, 2008.

Srinivasan, K. and Young, W. R.: Zonostrophic instability, J. Atmos. Sci., 69, 1633-1656, 2012.

Tanaka, Y. and Akitomo, K.: Alternating zonal flows in a two-layer wind driven ocean, J. Oceanogr., 66, 475-487, 2010.

Vallis, G. K. and Maltrud, M. E.: Generation of mean flows and jets on a beta plane and over topography, J. Phys. Oceanogr., 23, 1346-1362, 1993.

Wang, J., Spall, M. A., Flierl, G. R., and MalanotteRizzole, P.: A new mechanism for the generation of quasizonal jets in the ocean, Geophys. Res. Lett., 39, L10601, doi:10.1029/2012GL051861, 2012.

Wang, J., Spall, M., Flierl, G. R., and Malanotte-Rizzole, P.: Nonlinear radiating instability of a barotropic eastern boundary current, J. Phys. Oceanogr., 43, 1439-1452, 2013.

Zakharov, V. E. and Ostrovsky, L. A.: Modulation Instability: The beginning, Phyhsica D, 238, 540-548, 2009. 